\title{
Innovative Design of Household Coffee Maker for Chinese Market*
}

\author{
Xinghe Wang \\ College of Culture Communication \\ Liming Vocational University \\ Quanzhou, China 362000
}

\begin{abstract}
This paper analyzes the present situation and main problems of household coffee maker in Chinese marketas well as the consumption psychology of the target group and puts forward purposeful innovative designs which provide new concepts for the innovative design of household coffee maker for Chinese market by means of "Design Orientation - Design Concept and Design Details- Design Presentation".
\end{abstract}

Keywords-household coffee maker; Chinese market; innovative design

\section{INTRODUCTION}

In recent years, the household coffee maker market of China has changed quickly. On the one hand, because of the improvement of manufacturer's production technology, varieties and brands of the products are becoming increasingly diversified, leading to more intense competition and even price war sometimes[1]. On the other hand, consumers' consumption philosophy tends to be pragmatic, from the separate consideration of the product's function, brand, quality, etc. into comprehensive consideration on a variety of factors, such as cost performance, maintenance and so on. Individualization and customization of the products contributes to the diversified demand and complicated consumption. In this case, this paper provides a useful reference for the design of household coffee maker for Chinese market with a new design method.

\section{DESIGN ORIENTATION}

\section{A. Orientation of Target Group}

In today's fierce competition, household coffee maker, as a product for improving the taste of life and improving the aesthetic appeal, is necessary to accurately grasp the thinking of the consumers and understand their psychological needs. From the perspective of the enterprise, it must analyze the aesthetic preference of the target population in advance. Because of large market coverage, the consumer groups are not the same, and it needs to segment the market, select the appropriate target group and prejudge their potential social needs according to their lifestyle and consumption characteristics as the basis for the new product development.

*CLC No.: TB472 Document identification code: A

Foundation project: Quanzhou Social Science Planning Project (2017E18); Training Plan for Leaders in Young and Middle-aged Subjects (Major) of Quanzhou Institution of Higher Learning.
The consumer groups of household coffee maker in China are concentrated at present, mainly consisting of young people born after 70s, 80s and 90s.Because of the diversification of the beverages' consumer market, people born after 70s prefer Chinese and western combined beverages to tea. While people born after 80 s and 90 s are easier to accept new staff, most of whom have accepted coffee culture and increasingly treated coffee as day-to-day drinks.

\section{B. Analysis on Consumer Psychology}

The young people born after70s, 80s, and 90s have their own unique thinking and values, and they advocate personality and freedom, which lead to their fresher and more progressive consumption behavior, and the individualized consumption has become their inevitable choice [2]. Moreover, because they grow up under special background, their consumption characteristics are different showing pursuit of fashion, dare to consume and paying attention to the psychological and emotional satisfaction in the consumption experience, especially pursuing to the different, individual and diversified consumption experience[3].

\section{Design Orientation}

The design orientation of household coffee maker is summarized into four aspects as follows for the consumption demand of the targeted group: design on individual consumption, design on expectation of value for money, design on diversified demands and design on specific demands.

\section{DESIGN CONCEPT AND DESIGN DETAILS}

\section{A. Design on Individual Consumption}

1) Design concept: In view of young consumers characterized by fashion-pursuing and self-showing, the author participated in the design of a small household coffee maker, as shown in "Fig. 1", which is made by modular design principle that its main body, extracting tray and extracting mechanism are separable: the main body is shelled by plastic and metal envelope, which gives the product a sense of modern science and technology feeling while reduces the production cost, springing from the trapezoid with the features of simple, clear and orderly aesthetic feeling, and to avoid excessively stiff, an inner concave trapezoid opposite to 
the body direction is designed in the front face, symmetrically and full of changes.

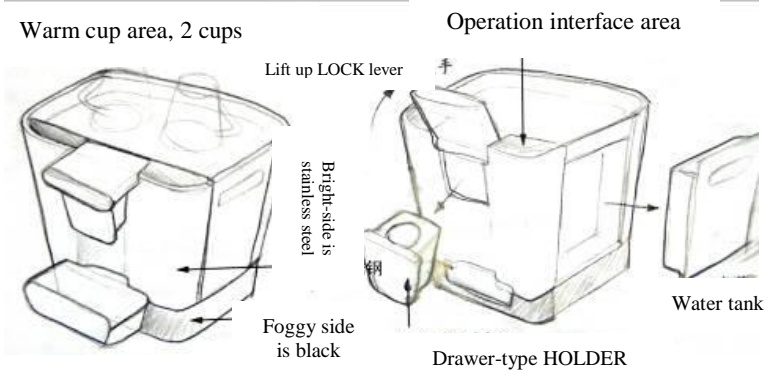

Fig. 1. Design concept of small household coffee maker.

2) Design details: The through-hole design of the extracting tray springs from the idea of checker, and the coffee extracting mechanism is of drawing type, easily operated as shown in "Fig. 2" Design details of small household coffee maker

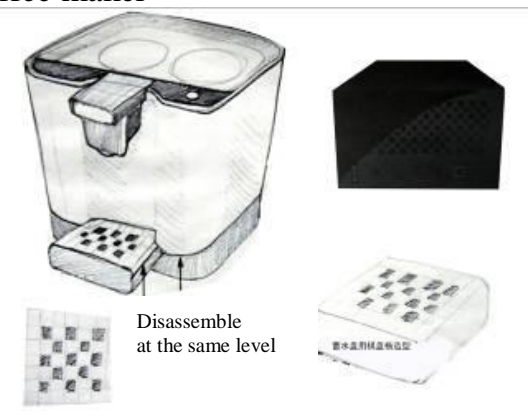

Fig. 2. Design details of small household coffee maker.

3) Design presentation: Finally, with the adoption of capsule extracting mode, the product avoids a large amount of professional operations, convenient and fast. In addition, by virtue of separable tray and extracting mechanism, they are easily washed and benefiting from its small size, it is also allowed being given as a gift together with capsule and cups, easily to carry and reduces the production cost and increase its cost performance, as shown in "Fig. 3" Design presentation of small household coffee maker.

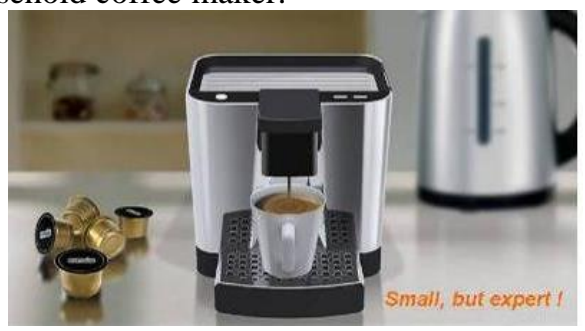

Fig. 3. Design presentation of small household coffee maker.

\section{B. Design on Expectation of Value for Money}

1) Design concept: The product is more and more diversified in the form of function, hence, it is always necessary for the customers to comprehensively consider the various functions of the product and achieve the value of money and even value for money. As shown in "Fig. 4", it is a

concept of more-in-one household coffee maker, co-designed by the author in EUPA Company, and this coffee maker is designed to simplify the complicated procedures of ice coffee making. Traditionally, the ice coffee needs to be added crushed ice after boiled, which not only makes it taste bad (the best serving temperature of coffee is $85^{\circ}$ ), but also requires coffee maker and crushed ice maker both. In view of this situation, the customers desire for a product perfectly combining with coffee maker and crushed ice maker that can save time and space while being cost effective.

A

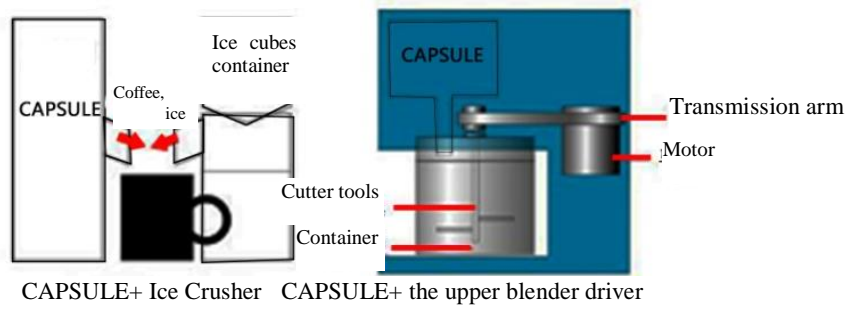

Fig. 4. Design concept of more-in-one household coffer maker.

2) Design details: For these two design concepts, the author selected the first mode and put forward new design concept, as shown in "Fig. 5" Design details of more-in-one household coffer maker.

\section{A}

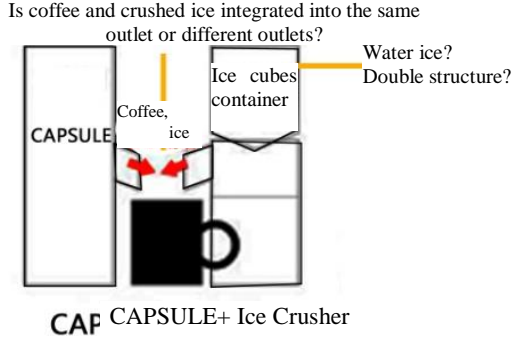

Fig. 5. Design details of more-in-one household coffer maker.

3) Design representation: After the design scheme and details are selected, the more-in-one household coffer maker is born as shown in "Fig. 6". It differs from the conventional coffee maker in that it is not only cable of exacting coffee or making ice cube separately, but also making two in one to make a quality ice coffee. Because it requires both water and ice cube tanks and at the same time keeping the product operate in stable condition (the product vibrates too much in ice crushing), so that the water tank and ice tank are designed to install at the top of the low-lying and bilateral area and uniformly set the control buttons on the metal panel for convenience and bringing out the best in each other. Because such extracting mode is the first time being provided, it has obtained the utility model patent. 


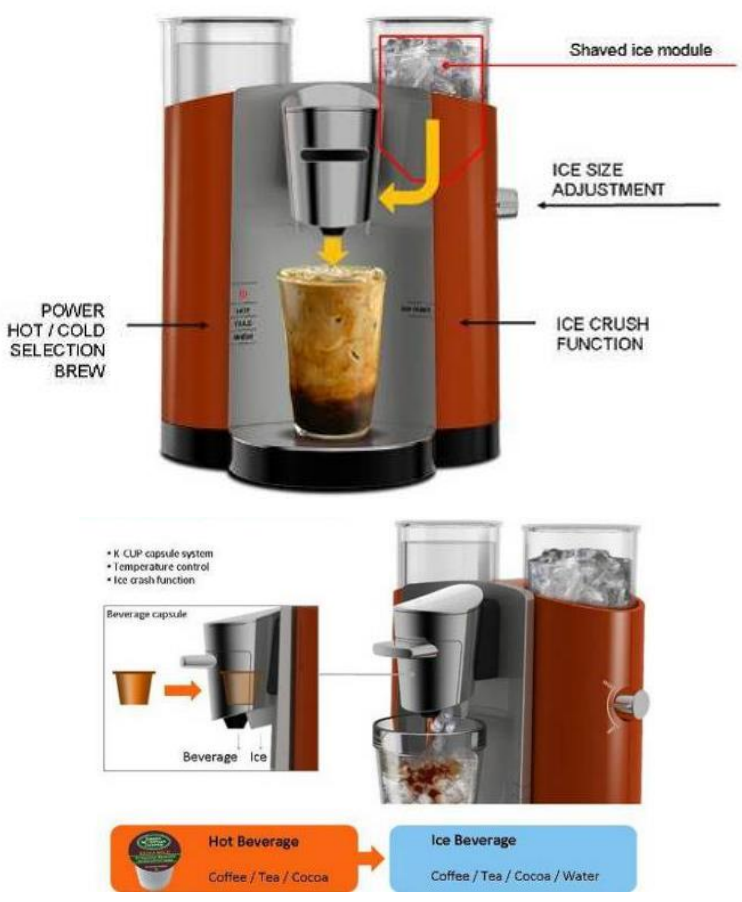

Fig. 6. Design representation of more-in-one household coffee maker.

\section{Design on Diversified Demands}

1) Design concept: The diversification of consumer demand leads to pluralism and diversification of the function of the household coffee maker. Fig. 7 is a sketch of milk foam machine co-designed by the author in EUPA Company. Milk foam to coffee is what salt to meal, which is necessary in making the current dominant coffee, e.g. espresso, Latte and Cappuccino to some extent as an important part. The making of traditional milk foams is relatively difficult that needs other auxiliary equipment and strong professional experience. In view of this problem, based on the actual design needs of the company, the author designs some sketches of milk foam machines as follows.

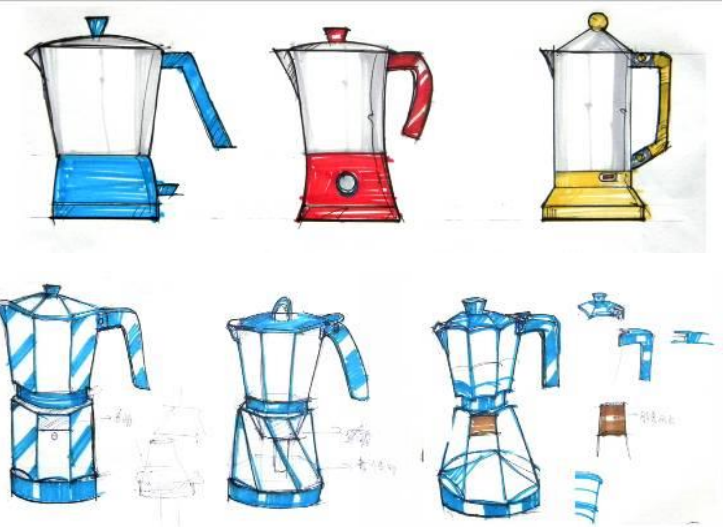

Fig. 7. Design concept of milk foam machine.

2) Design details: In view of the sketch above and according to related suggestions, the author selected the design sketch in the middle and adopted Corel to define the 2D size as shown in "Fig 8". Design details of milk foam machine.

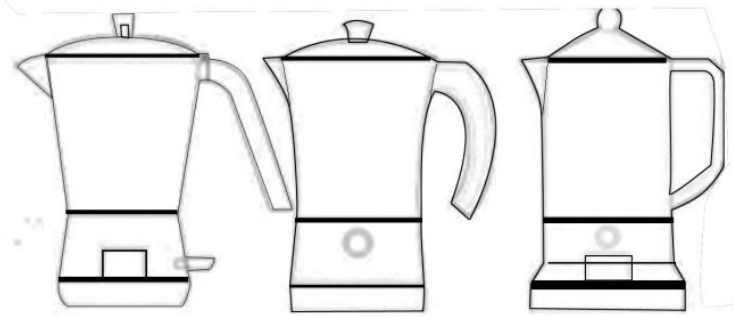

Fig. 8. Design details of milk foam machine.

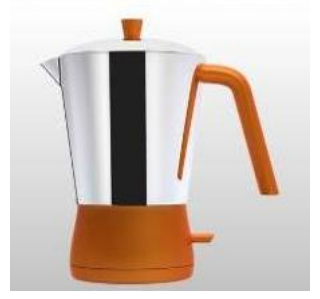

Fig. 9. Design representation of milk foam machine.

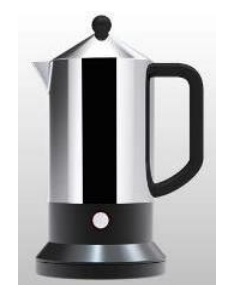

Fig. 10. Design representation of milk foam machine.

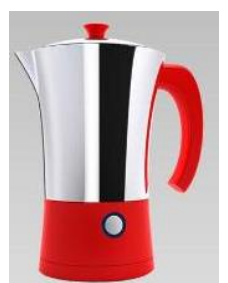

Fig. 11. Design representation of milk foam machine.

3) Design representation: After the $2 \mathrm{D}$ size is defined and adjusted, the author designed the series of milk foam machine as shown in figure 9,10 and 11 , characterized by the following: one-touch operation, heating while foaming milk, auto power off upon temperature reaching $70^{\circ} \mathrm{C}$; magnetic pole adopted, with high power utilization. Because of the adoption of the same functional principle, the internal components of the product are consistent, the design period can be shortened and the production cost is reduced, while the diversified products can effectively meet different individual needs of the customers.

\section{Design on Specific Demand}

1) Design concept: "Fig. 12" is a sketch of household mini-mocha pot for Bialetti co-designed by the author in 
EUPA Company, which can, with the adoption of capsule extraction, attract the traditional Mocha pot enthusiasts with the unique image and structure of the Mocha pot, arousing their emotional resonance, so as to increase the market share.

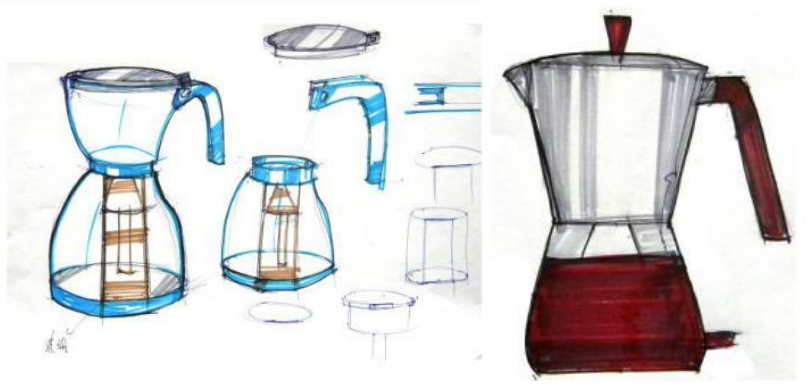

Fig. 12. Design concept of household mini-mocha pot for Bialetti.

2) Design details: In view of the sketch above and according to related suggestions, the author selected the last design sketch and adopted Corel to define the 2D size and carried out detail adjustment as shown in "Fig. 13" Design details of household mini-mocha pot for Bialetti.

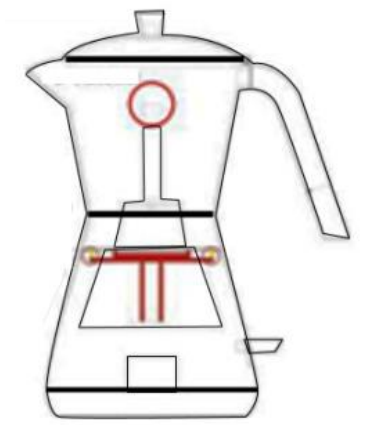

Fig. 13. Design details of household mini-mocha pot for Bialetti.

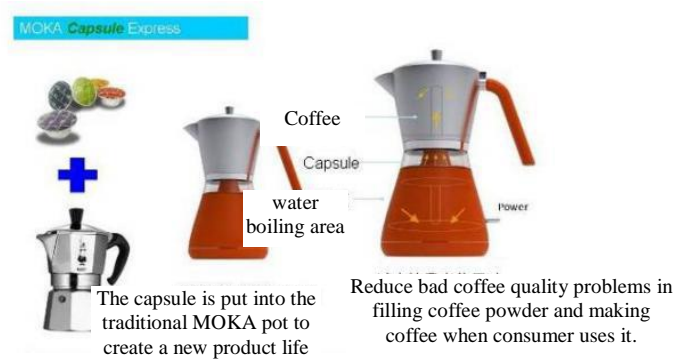

Fig. 14. Design representation of household mini-mocha pot for Bialetti.

3) Detail representation: After the $2 \mathrm{D}$ size is defined and adjusted, the author designed the household mini-mocha pot as shown in "Fig. 14", which can, with the adoption of capsule extraction, solve the problems of inappropriate filling of coffee power and low coffee quality in use. In the meanwhile, it employs one-touch operation that is free from additional manual operation from coffee extraction to the end, which extremely expresses its user-friendly design.

\section{CONCLUSION}

This design is indeed to create a living environment that is more suitable for people's life, and its service object is human. To be accepted, loved and even enjoyed, it must have a resonance and interaction with user. The design of the household coffee maker for the Chinese market is to define the target group and analyze their consumption psychology and demand under the guidance of the people-oriented thought, and then put forward the design orientation of the product in combination with the specific project and production feasibility of the company, and further carry out design concept and details step by step, seeking to achieve the standardization of the design procedure.

\section{REFERENCES}

[1] Wang Xinghe, Exploration and Analysis on the Trend of Household Coffee Maker for Chinese Market [J].Journal of Chifeng University(Natural Science Edition), 2015, 31 (11) :63. 王星河.国内家 用咖啡机设计趋势探析 [J]. 赤峰学院学报 (自然科学版), 2015, 31 (11) : 63

[2] Lin Feng, Cheng Lihua. Research on Design of Younger-generationtargeted Product [J].PACKAGING ENGINEERING, 2010, 31(20):35.

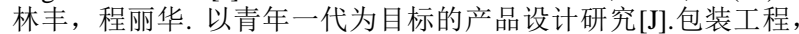
2010, $31(20): 35$.

[3] QuJunlin. Brief Discussion on Innovation and Developing Path of Industrial Design [J].Manager'Journal, 2016 (3) :201. 曲俊霖.浅谈工业 设计创新和发展路径[J].营管理者, 2016 (3) : 201 . 